\title{
Does Hepatitis E Virus Need to Be Considered as a Re-Emerging Problem in Both Industrialized and Developing Countries?
}

\author{
Ziyue Zhang ${ }^{1}$, Tong $\mathrm{Wu}^{2}$, Miranda Zhong ${ }^{3}$, Jiawen Xiao4, Ziyu Dong5 \\ ${ }^{1}$ Beijing No. 101 Middle School, Beijing, China; ${ }^{2}$ Suzhou Singapore International School, Suzhou, China; ${ }^{3}$ BASIS \\ International School, Shenzhen, China; ${ }^{4}$ Arrowhead Christian Academy, Redlands, CA, United States; ${ }^{5}$ Wuhan \\ Institute of Biological Engineering, Wuhan, China
}

Correspondence to: Ziyue Zhang, 18612746176zzy@gmail.com

Keywords: Hepatitis E Virus, Virology, Transmission, Prevention, Treatment

Received: September 11, $2018 \quad$ Accepted: October 23, $2018 \quad$ Published: October 26, 2018

Copyright (๑) 2018 by authors and Scientific Research Publishing Inc.

This work is licensed under the Creative Commons Attribution-NonCommercial International License (CC BY-NC 4.0).

http://creativecommons.org/licenses/by-nc/4.0/

(c) (i) (9) Open Access

\section{ABSTRACT}

Hepatitis E caused by the Hepatitis E Virus (HEV) is a liver disease resulting in over 20 million cases every year. Hepatitis $\mathrm{E}$ is now considered by some scientists as an emerging issue as HEV is not only prevalent in developing countries but is increasingly detected in industrialized nations. In this paper, we try to provide evidence for this notion and what actions may need to be implemented to prevent further spreading of HEV. The prevalence of HEV, including its distinct genotype distribution in different geographic regions (including both developing countries and industrialized countries) will be discussed; further discussions of HEV treatments will include the availability and the mechanism of HEV vaccines and antiviral treatments used to treat and contain the disease. Additionally, the prevention and spreading of Hepatitis $\mathrm{E}$ disease will be discussed in the later section of the paper. With the presentation of HEV transmission route, infection regions, and treatment, we aim to raise the awareness of the general public toward this liver disease and discuss whether the high prevalence disease is an emerging disease worldwide. According to the data we collected, the rate of HEV infection is high and shows a trend of increasing, which leads to our conclusion and proves our hypothesis that Hepatitis $\mathrm{E}$ is a re-emerging disease.

\section{INTRODUCTION}

In 1978, in the Kashmir Valley of India, an unprecedented outbreak of a water-borne disease swept around the area and resulted in over 52,000 infected cases, including 1700 deaths, raising the attention of 
the local authorities $[1,2]$. This unknown phenomenon drew up a high public health concern and subsequently led to intensive studies focusing on identifying the cause of these symptoms. The clinical symptoms of patients were similar to those of Hepatitis $\mathrm{A}$, but symptomatic individuals did not have antibodies for both Hepatitis A Virus (HAV) and Hepatitis B Virus (HBV). These observations suggested that patients were suffering from a new "enteric non-A non-B Hepatitis (ENANBH) form". However, little was known about this disease until 1983, when the etiologic agent was successfully isolated by Russian scientist Mikhail Balayan. He conducted a self-experiment ingesting a stool sample from infected soldiers, allowing him to transfer the disease back to his laboratory [3]. Similarly, larger outbreaks emerged in India, China, and Germany and killed thousands of people. Through autopsies, scientists discovered that all the patients infected with this virus had severe liver damages; hence, the virus was later classified as the Hepatitis $\mathrm{E}$ Virus (HEV).

\subsection{Hepatitis E Disease Description \& Symptoms}

Ample epidemiological surveys testing for the presence of anti-HEV antibodies have been performed, all of which demonstrate that large numbers of people have been exposed to HEV at some point in their lives; however, HEV does not always provoke clinical apparent symptoms [4]. Early signs of HEV infections, such as fever, vomiting, and faintness, are usually ignored or misinterpreted as signs of the common flu; more characteristic symptoms, including but not limited to jaundice, itching, pale stools, and darkened urine, are shown in the terminal stage of infection, and also correlate with chronic liver damage [5].

Two stages of Hepatitis E are detected, including acute and chronic phase. Generally, patients who are affected by acute infection do not proceed to the chronic phase. People who have immunodeficiencies or received immune suppressants e.g. due to organ-transplantation are prone to develop chronic Hepatitis $\mathrm{E}$. Pregnant women also exhibit particularity severe symptoms; their unique immune features make them incapable to receive most of the clinical drugs; consequently, a high mortality rate around $30 \%$ is disclosed among the infected pregnant women [4].

\subsection{HEV Transmission}

The main HEV transmission route is through food consumption-consumers who ingest infected food and then get infected. HEV can also be transmitted through the fecal-oral route, meaning that pathogens in fecal particles would pass from one person to the mouth of another person [5]. Due to the multiple transmission route of HEV, it is crucial to minimize the intake of uncooked foods (it has been proven that $\mathrm{HEV}$ in raw animal products can be eliminated by cooking above 70 degrees Celsius for 20 mins). WHO has stated that there is a drastic increase of the Hepatitis E positive population in Germany in the last decade-the annual rate of endemic cases increased from $30 \%$ to approximately $78 \%$ [4].

Blood-transfusion and organ-transplantation are the other two transmission pathway. In one of the reported cases, the patient who received full blood plasma transfusion, got infected by HEV, thus, developed into chronic HEV due to the immune suppressants he took, which led to a further damage to his health condition. The blood transfusion-associated HEV appears globally in recent years among European countries such as Germany and France, Asian regions such as Japan, and North American countries such as the United States [4].

The underdeveloped sanitary facility and the unqualified hygiene standard establish the fundamental condition that triggers the outbreak of HEV. Subsequently, rate of HEV infection is extremely high in developing countries. In short, the primary task is to improve the environmental conditions in those underdeveloped areas [4].

\subsection{HEV Molecular Virology \& Heterogeneity}

There are currently 8 known HEV genotypes (parts of the genetic makeup of a cell that determines the phenotype of a specific organism). It has previously been shown that genotypes 5,6 , and 8 are only infectious to animal species whereas genotype 1 and 2 are specifically infectious to human. Studies have 
suggested that genotype 3 and 4 could be spread to both humans and animals through different routes. [6] Current research has shown evidence of infection of genotype 7 on camels and only one case of infection on humans; hence, more research needs to be constructed for the indication of susceptible species of this genotype [7].

HEV is a non-enveloped, single-stranded, positive-sense RNA virus which presents three Open Reading Frames (ORFs) in its genome: ORF1, ORF2, and ORF3. ORF1 occupies two thirds of the HEV genome and encodes for non-structural proteins of domains of methyltransferase, papain-like cysteine protease, RNA helicase, and RNA-dependent RNA polymerase activity of the virus; ORF2 encodes for the viral capsid protein, and it overlaps with ORF3; ORF3, shown in recent research, serves as viroporin and encodes for the ion channel that facilitates ion influx and outflow of the virus $[8,9]$.

Although scientists are now able to propagate HEV in laboratories, the life cycle of the virus remains incompletely understood (Figure 1). However, it is believed that HEV enters the host cells by binding to

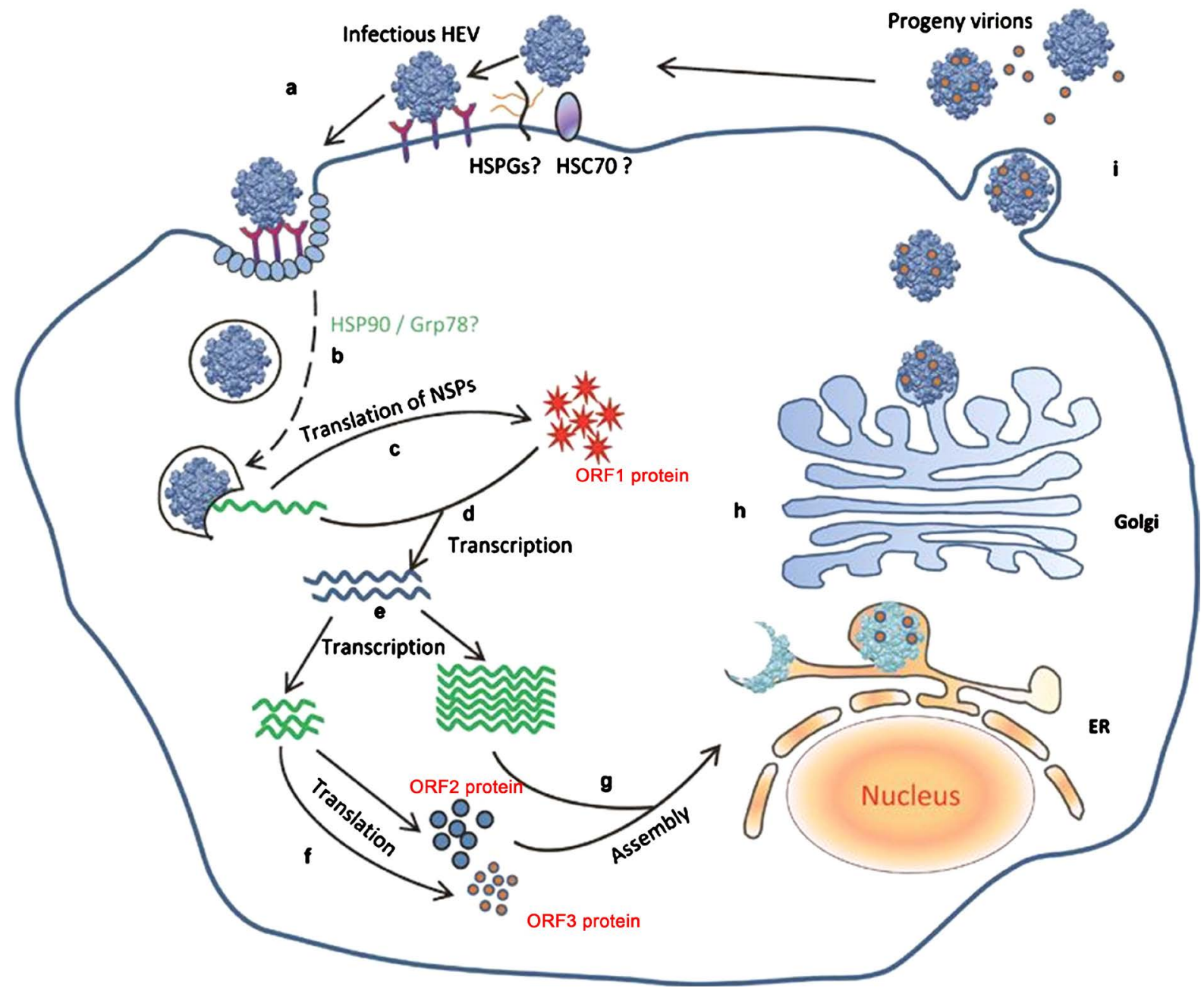

Figure 1. Proposed Life Cycle of HEV. Step a: HEV attaches to cell surface via HSPGs or HSC70 and then enters the cell through unknown receptor. Step b: HEV penetrates the cell membrane and enters the cell. HSPGs and HSC70 may be involved in the process. Step c: the positive-sense genomic viral RNA serves as the template of translation of ORF1 proteins. Step d: The viral RdRp synthesizes an intermediate, replicative negative-sense RNA. Step e: transcription of viral RNA. Step f: ORF2 and ORF 3 are translated. Step g: assembly of HEV virus. Step h: the nascent virus is transported to the cell membrane. Step i: the virus is released from the infected cell (Dianjun Cao, et al. 2012) [10]. 
cellular receptors with its capsid proteins allowing the virus to enter the host cell, then the viral genome is released into the host cell, the ribosome is recruited to translate viral RNA into viral proteins. The process of resembling and release of Hepatitis E virus is partially comprehended; hence, advanced researches in the life cycle of Hepatitis E virus are necessary. As by the further comprehension of the life cycle of the virus, scientists may eventually find a way of treating or preventing HEV infection by interfering the process of replication of the HEV [10].

HEV is now spreading globally and becoming an immediate emerging problem due to its specific transmission route and various genotypes. Through intermediates such as uncooked meat or contaminated water, healthy individuals would then be infected by HEV, resulting in a consequential effect in a larger cycle. In different geographic regions, different HEV genotypes are prevalent: The outbreaks of genotype 1 and 2 mostly occurred in developing countries, whereas outbreaks of genotype 3 and 4 are more commonly discovered in developed countries (e.g. Gt 1, 2 in China and Gt 3 in Germany); hence, the virus is diverse among its hosts, which increase the difficulty in controlling the spread of the virus [11]. However, due to its limited treatments, alternatives are desperately needed to replace RBV and IFN. It will be necessary to contain HEV more efficiently viral transmission, possible ways including screening blood supplies, assuring food safety and more wide-spread use of the HEV vaccine. In this paper, questions related to the current situation of hepatitis $\mathrm{E}$ will be further discussed, including prevention, treatments, and the problems researchers encounter within current studies of HEV.

\section{HEPATITIS E INFECTIONS IN BOTH INDUSTRIALIZED AND DEVELOPING COUNTRIES}

HEV is prevalent in many countries. The main routes of HEV transmission vary, however, significantly in different geographical regions. Our further analysis is based on the different situation facing by countries such as China, Germany, and India, illustrating the variety of HEV infection in developed countries and developing countries.

\subsection{Infection in Germany}

Hepatitis $\mathrm{E}$ has been an under-reported infectious disease in Germany until recently. 17\% of the Germany population was once infected with HEV and this infection rate has been increased for at least 40 folds in the past decades, subsequently, gaining publicity and health concerns within the country. A primary factor that contributes to the spread of HEV is eating uncooked pork products, which is fairly popular among the Germans. It has been identified that four wild boar populations in east and west Germany are also reservoirs of HEV genotype 3 and present high rate of HEV infections [12, 13].

HEV within pork products cannot be eradicated if uncooked. In a research conducted in 2001, 200 porcine liver samples in 81 butcher shops and groceries collected from all across Germany were tested, and 4 of the 200 samples were contaminated with HEV. Further research showed that 14 out of $70(20 \%)$ of the tested raw sausages and 11 out of 50 (22\%) of the liver sausages were HEV RNA positive [14].

The consumption of these pork products will lead to a wide range of HEV infection in humans, in fact, it has already contributed to 300,000 cases per year nationwide [12].

People who live in close contact with pigs daily, usually pig farmers and swine hunters, are especially susceptible to HEV infection. Statistical records comparing the seroprevalence of subjects in contact with pigs $(13.2 \%-32.8 \%)$ to that of non-exposed individuals $(7.7 \%-21.7 \%)$ shows a significant difference [15]. Hence, the focus of controlling HEV infection rate in industrialized countries such as Germany should be on the screening of pig herds for swine HEV infection and vaccination of susceptible people. The latter would require, however, the approval of the Chinese HEV vaccine to the European market.

In addition to the transmission of HEV from infected swine to humans, human to human transmission is another route of spreading viral infection. Human to human transmission of HEV in Germany is occasionally caused by blood transfusion-people who receive blood donation from HEV positive patients generally get infected by the virus and develop Hepatitis E. In Germany, the first recorded case of transfusion-associated HEV infection was in 2013. The patient was tested HEV RNA positive and suffered from 
chronic HEV infection after receiving aphaeresis platelets from a blood donor on July $4^{\text {th }}, 2013$. The donor was tracked down and tested, and the result showed that he was infected with HEV during the blood donation but did not show any initial symptoms [16]. This specific incidence raised public awareness towards the safety of blood transfusion. 69 in 1019 blood donors (6.8\%) tested are anti-HEV IgG positive, meaning they were once infected with HEV. Later, a sample of 16,125l blood donors was screened during donation, about $0.08 \%$ of which is tested HEV RNA positive, indicating that around 20,000 blood donors will transmit HEV to patients receiving blood transfusion [17, 18]. Although no vaccine is available in Germany to prevent transfusion-associated HEV infection among patients, the method of screening blood pools with viral RNA is now under research.

\subsection{Infection in India}

India has long been an endemic zone of enterically transmitted viral hepatitis. Recent research shows the HEV prevalence in different regions of India: 53\% in Kashmir, 9.4\% - 58.9\% in Uttaranchal, $44.6 \%$ in Punjab, 53.3\% in Delhi, 17.9\% in Uttar Pradesh, $42 \%$ in Bihar, $41.05 \%$ in West Bengal, $49.7 \%$ in Rajasthan, $35.76 \%$ in Mumbai, and $17.3 \%$ in Chonnal [19] (Figure 2).

However, unlike Germany, the main transmission routes of HEV in India are not porcine or transfusion-associated. HEV is transmitted through sewage-contaminated water in India due to their low sanitation standard. About $80 \%$ of the sewage generated flows into the main water resource: natural rivers, lakes, ponds, and the underground water [20]. A recent study showed that in Northern India, 79 out of the 192 (41\%) sewage samples were HEV RNA positive, and in Southern India, 80 out of 144 (55.6\%) were tested positive [21]. As mentioned in the previous sections, HEV can be transmitted through the fecal-oral route, Hence, it is reasonable to conclude that most cases of HEV infections in India are caused by consumption of contaminated water. Therefore, the main focus has to be on ensuring clean water supplies across the continent to limit the spread of HEV. Additionally, mass vaccination could further aid in spreading HEV. However, as stated numerous times, the HEV vaccine is currently only available in China.
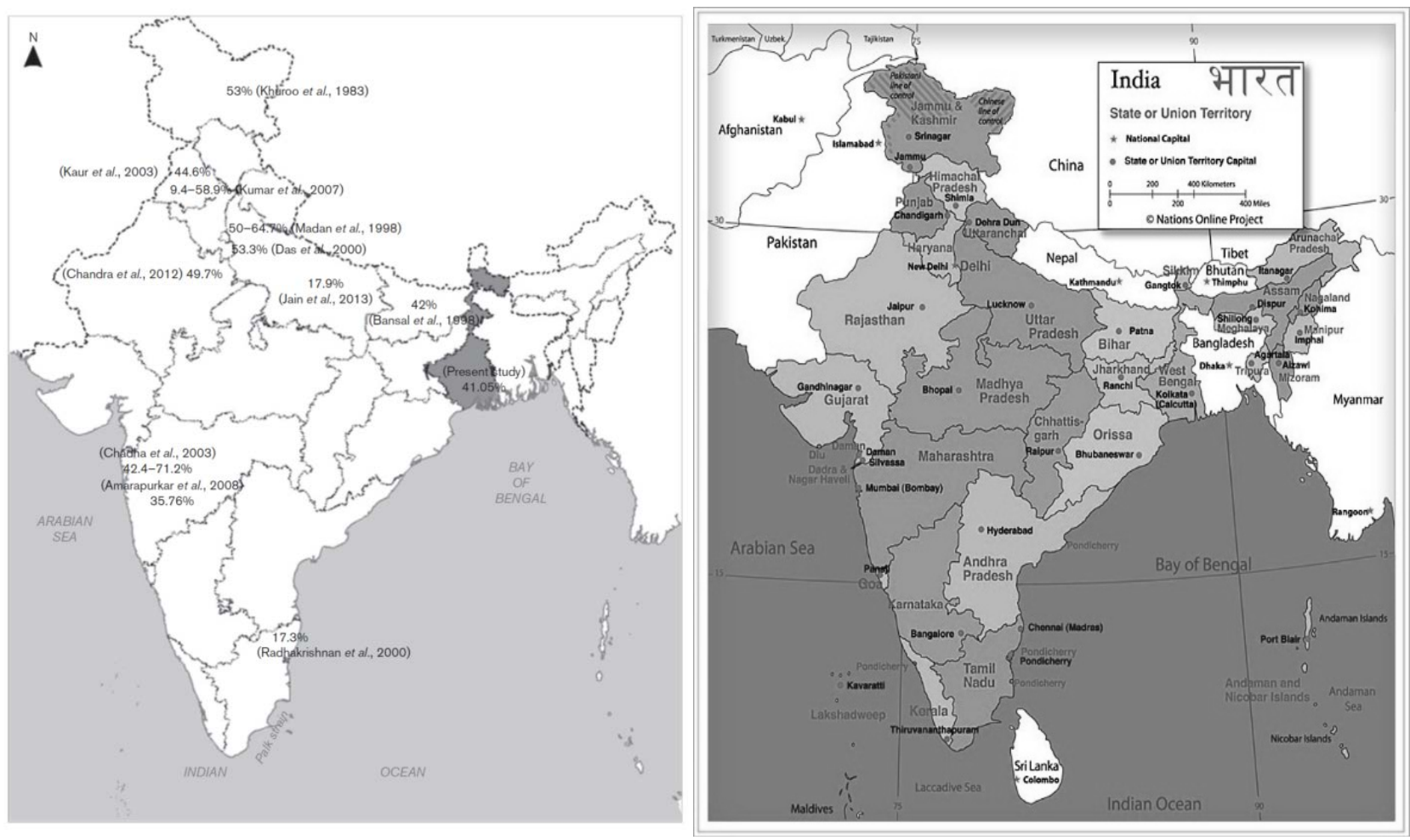

Figure 2. Rate of infection of HEV in different regions of India (Jain P., et al. 2013) [19]. 


\subsection{Infection in China}

The infection rate of HEV in China varies by the disparity between rural and urban areas. Low quality of hygiene and consumption of contaminated food is the main cause of HEV transmission in the rural areas. Tibet, for example, a typical representative of rural regions in China, develops a high infection rate of HEV genotype 3 and 4 due to the nomadic lifestyle adopted by the locals who rely on the domestication of livestock as a financial resource. A recent study has shown that 236 of the 600 individuals, i.e. approximately 40 percent, in a study cohort from that region present positive results for anti-HEV IgG [22]. In contrast, some of the more developed areas in China such as Anhui, Beijing, and Henan, the infection rate ranges from 10\% - 20\%; in Jiangsu, Chongqing, and Shanxi 20\%-30\% of the whole population has been exposed at some point in their lives to HEV [23]. The detailed infection rate in percentage categorized by districts of China is listed below in the pie chart.

Another factor contributing to the overall fairly high HEV prevalence in China is the large consumption of pork. Over 400,000 tons of pork is imported to China every year. Furthermore, research has shown that HEV prevalence among pig herds in different regions of China is relatively high, ranging from $1.6 \%$ to $37.5 \%$ [23]. However, the safety of pork products, irrespective of whether they are domestic or imported, cannot be guaranteed: there is an underlying risk of being contaminated with HEV (primarily HEV genotype 3 and 4), which likely contributes to spreading the disease to humans nationwide. One possibility to break the transmission cycle would be to vaccinate more people or even consider veterinary vaccinations. However, the latter is impractical when considering the significant costs of vaccinating entire pig herds of millions of animals.

\section{HEPATITIS E DISEASE CONTROL \& PREVENTION}

\subsection{HEV Vaccine}

HEV vaccine is made up of a type of HEV capsid-derived protein antigen in conjunction with an adjuvant [25]. Its mechanism is hinging on the function of ORF2, encoding for capsid protein involved in virion assembly and virus' immunogenicity, and infectious particles composed of three glycosylation sites could be formed when the corresponding protein is transcribed [26].

Multiple studies were then directed to assess the immunogenicity and efficacy of the HEV vaccine. In a clinical trial conducted in 2007, 2000 out of the 5323 investigated subjects, who were at risk of contracting HEV, were involved in the experiment to determine the efficacy of the HEV vaccine. Within the testing group excluded from the dropout, 1000 randomly selected subjects were immunized and another 1000 received a placebo. Then, each sample is measured for anti-HEV immunoglobulin that affiliates the reciprocal antigen. The result showed that the immunoglobulin found inside the vaccinated group increased to $100 \%$ till the third dose; by the time that the trial ended, their immunoglobulin count has decreased to $56.3 \%$. Meanwhile, in the placebo group, the HEV antibody presented increased to $10.6 \%$, which indicates infections of HEV (Figure 3) [27].

The chart (Figure 4) illustrates the HEV antibody percentage in respond to days during clinical trial, a measurement of antibody was conducted after each dose. A significant increase of antibody in vaccine group could be seen after the third dose. The slightly increase of antibody in placebo group may indicates the infection of HEV [27].

The final conclusion was drawn from the measurement of anti-HEV immunoglobulin that affiliates the reciprocal antigen. Hence, this vaccine was proven to be effective. Fortuitously, this outcome shows that other regions would yield the same effects since all HEVs are generated from one single serotype. Another long-term efficiency test of the HEV vaccine shows that in order to prevent people from HEV infection in a long run, at least three doses of such vaccine need to be injected, and after these three doses of vaccine injected, full immunity towards HEV will be conducted [28].

While the clinical trial reflects that this vaccine is effective, the HEV vaccine is only available in China. However, HEV vaccination in China is not compulsory. Although there are conclusive data on how many people in China have been vaccinated, a survey conducted by a group of Chinese researchers attests 


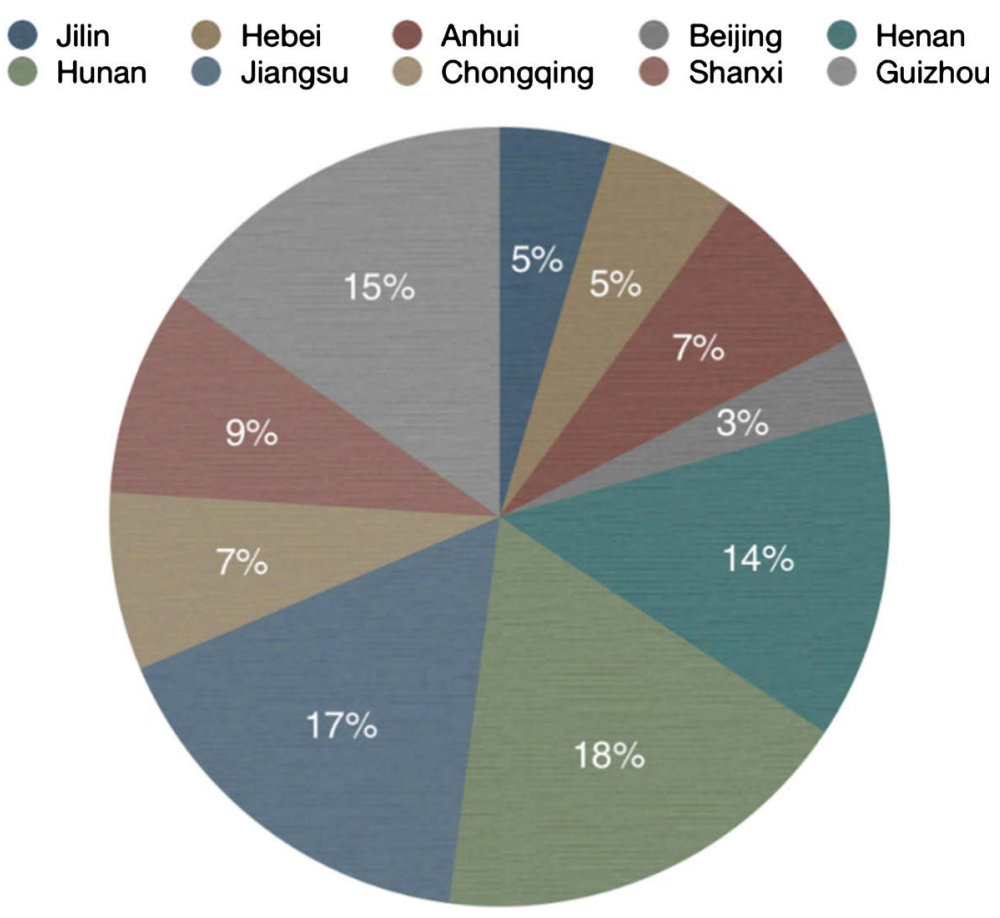

Figure 3. Seroprevalence of Anti-HEV in 11 provinces, China (Dong, et al. 2012) [24].

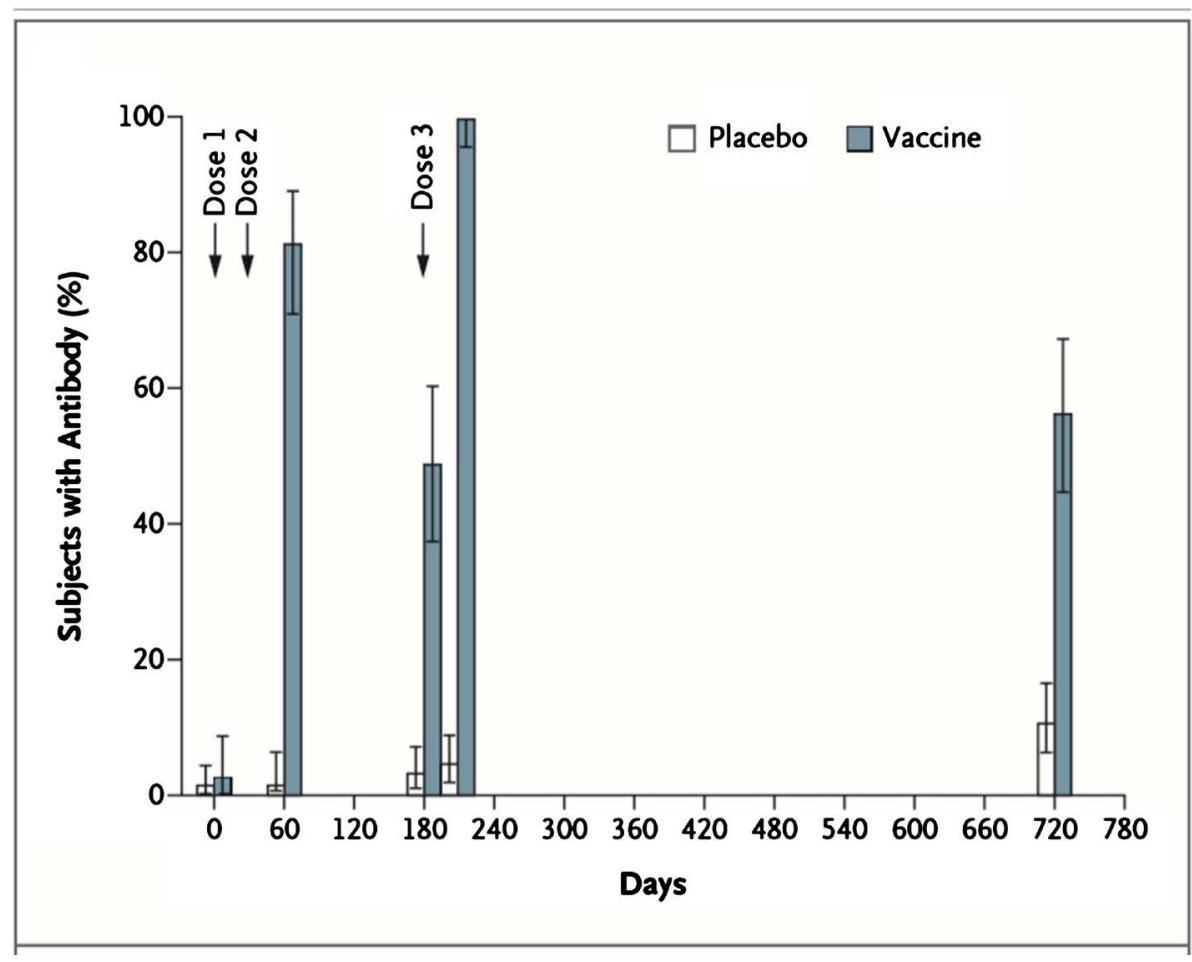

Figure 4 . HEV antibody percentage after vaccination.

that $82.49 \%$ of the investigated population are willing to pay for such vaccines [29]. This indicates a great prospect of this vaccine in high risk areas.

Practicality of Implementing the HEV Vaccine in Industrialized Countries

On the bright side, the prevalence of such vaccine is shown to be practical, especially in highly in- 
fected developed countries in Germany. Assuming each county is in equilibrium, one could therefore use GDP per Capita PPP, GDP per capita calculated based on purchasing power parity, as an indicator as average annual income in a given country, providing an impartial reflection of punching ability in the discussed countries. Of the three previously discussed countries, Germany has the highest purchasing power in 2017 (44,432 USD), followed by China (14,401 USD) and India (6096 USD) [30, 31]. This statistic shows that the dissemination in Germany and China should be relatively easier, since the family has more excrescent income used for family health care. For countries that have the condition to implicate vaccination to pigs and other susceptible animals should create standardized examinations before the meat has been imported inside the market.

\section{Practicality of Implementing the HEV Vaccine in Developing Countries}

As for India, other preventions that interrupts with HEV's transmission cycle could be implemented, such as proclaiming the consumption of well-cooked meat cuisine, or improving their hygiene by constructing more drainage and filtration system in the condensed population areas. Especially the latter approaches may be particularly important since sewage is the main route of HEV transmission [32]. What is more, the local government should take the risk of potential HEV outbreaks more seriously. The administrator from the government needs to provide subsidies to vaccine researchers and producers to abate its market price if needed.

Last but not least, the last threshold must be implemented in order to fully eliminate HEV transmission, which means vaccination for recipients prior to transplantation and subsequent immunosuppression. For instance, recommendation of vaccination shall be made to those patients who are about to receive organ transplantation. If one is informed that he or she will need an organ transplant at some point, it's better for them to get vaccinated before the surgery. Statistics from the organ donation administration centers of each countries shows that, in Germany, there are 797 cases of recorded successful organ transplanted in 2017, whereas there are 5148 cases recorded in China $[33,34]$. This implies that the demand of such vaccines from organ transplant patients in China is much higher than those in Germany.

\subsection{Blood Screening}

The transmission diversity in HEV with an increasing infection rate indicates the importance of HEV prevalence being assessed in blood screenings. In industrialized countries (China and Germany included), blood samples are usually selected for anti-HEV IgM or IgG testing before donation $[17,35,36]$. Conversely, no definite blood screening policy is set in HEV screening in India [37] and common assays like PCR in blood HEV RNA is also rarely practiced due to their lax blood screening policies; a study of HEV blood testing assays are conducted in different geographical regions for research purposes. In a Chinese study, a high viremia prevalence ranging from $0.1 \%$ to $29.2 \%$ among the blood samples tested for anti-HEV IgM and IgG, and the viral antigen HEV RNA is presented [38]. An increase of HEV prevalence was observed in Germany in 2014 to 2015 from 670 to 1267 [12]; therefore, the establishment of blood screenings are necessary in both developed and developing countries, since cirrhosis and severe liver damages may be developed after the acute infection; in some epidemiological areas like Punjab in India, routine examinations should be conducted in blood centers to prevent further spreading of the disease.

Possible prevention could be conducted by using serological screening for anti-HEV IgM in blood donors, reducing transfusion-transmitted risks especially for immunocompromised individuals and pregnant women $[4,39]$. Moreover, a routine HEV antibody testing could be performed among blood donors to control the prevalence of HEV. In recent years, HEV-antigen testing paper was invented (WANTAI BioPharm, China), which uses the "enzyme-linked immunosorbent" assay, primarily focusing on the IgM within the patient's body and providing instant results [40]. Moreover, the price for the testing paper is approximately $\$ 25$ per 1000 testing papers [41], which is a reasonable cost that the blood donation organizations are able to afford; hence, it is possible to generalize the HEV-antigen testing paper usages in blood centers and clinics and add it to blood testing to effectively monitor HEV, to those people who tested positive could be further tested and receive treatments in time if needed. 
Routine blood screenings are necessary for the control of HEV transmission and prevalence, and it should be used in more countries to address the global health concern; however, there might be some underlying difficulties that blood centers could face, including the size of sample pool and limited financial support in addition to the unlikeness of testing blood the samples used in an emergent time like transfusions (the time needed for PCR and the sample size).

Ranging from $4.10 \%$ to $27.9 \%$, the seroprevalence of HEV showed in German study has indicated is higher in humans living in area with a lot of pig farming in Germany [15]. The group of individuals who are exposed to easily-infected and infected species have a higher risk of getting infected by HEV. Considering the limited treatments, blood screenings should be used advocated specifically corresponding to the individuals who have high-risk in epidemic areas as well as transplant or blood donation recipients; Nevertheless, to achieve the full control of this zoonosis (HEV), it is necessary to address the safeties of the domestic pigs and other animals entering the human food chain.

\subsection{Treatments}

Studies have shown that pregnant women and those who are immunocompromised, usually those who received organ-transplantation, are the most vulnerable to HEV infection. Unfortunately, they are also the ones who are not able to receive existing treatments, such as ribavirin (RBV) or interferons (IFN), since RBV is teratogenic and IFNs can lead to graft rejection [42]. Thus, alternatives to these drugs need to be developed.

The most common way of treating HEV infection is by using the antiviral therapy, which includes the usage of RBV or IFNs [4]. IFNs are cytokines induced by the immune system usually in response to infection [43]. There are generally three classes of IFNs, type I, II and III. In this case, type I IFN, specifically a subtype called IFN $\alpha$ is used against HEV. IFN $\alpha$ was shown effective in treating Hepatitis E (research showed that $95 \%$ of HEV was eliminated in the observed patient) [44]. To increase the half-life of exogenously administered IFN, a pegylated form (pegIFN $\alpha$ ) has been developed which is highly effective in abrogating HEV (100\% in the sixth month) [45]. More specifically, before the patients have intake the $\mathrm{RBV}$, they should first wait for a few months no matter whether their immunological risk is high or low and then see if their bodies could immunologically fight the pathogen themselves. If their infections do not get any better after these months, an antiviral therapy using ribavirin will be needed. Then, if further testing shows that the HEV RNA still test as positive, then the ribavirin therapy should be continuously proceeded. Otherwise, the patient could continue the treatment after the HEV relapses (Figure 5).

However, treatment with IFNs has substantial side effects and can increase the risk of graft rejection and thus their practical utility is limited. The side effects of RBV include hemolytic anemia, pulmonary disorders, hypersensitivity and even bone marrow suppression [42]. Furthermore, cardiac patients, pregnant women, and their male partners should not be taking RBV, as a reverse effect in treating HEV may be established, which further lead to side effects like hypertension, birth defects and even stillbirth [5, 47].

\section{CONCLUSION}

Based on our research, we can draw on the conclusion that Hepatitis E can be now identified as a re-emerging disease and is causing millions of cases worldwide every year. HEV is highly prevalent in both many developing and industrialized nations and significantly contributes to human morbidity and mortality world-wide. Thus, raising public awareness to the prevalence of Hepatitis E is necessary. Further, generation of novel treatments that suit all groups of people (including pregnant women) and methods of prevention that can be applied in all countries must be a priority. As mentioned in previous sections, the current treatment for Hepatitis $\mathrm{E}$ includes the use of ribavirin and interferons which have proven efficacy. However, both treatments can cause severe side effects and cannot be employed when treating pregnant women infected by HEV and chronic Hepatitis E in immunocompromised transplant recipients; thus, there is an urgent need for developing novel, more tolerable treatments. Furthermore, actions in preventing HEV from spreading should be taken. Since there are great differences in the cause of HEV infections 


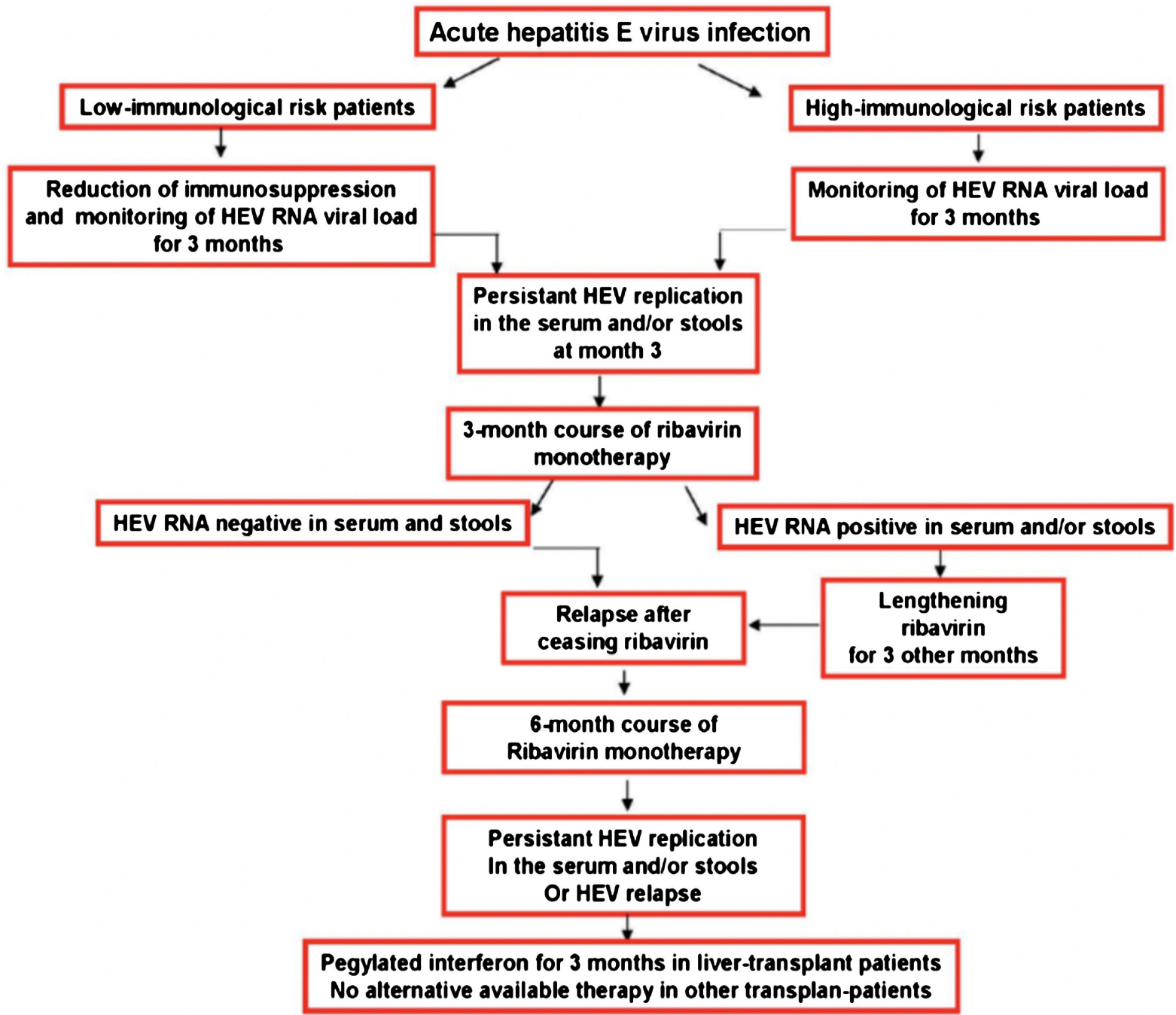

Figure 5. Simplified procedure of treating acute HEV infection Kamar, N., Lhomme, S., Peron, O. M., Alric, L., \& Izopet, J. (2016, August 15) [46].

between industrialized countries and developing countries, preventive strategies need to be considered based on separate situations. In industrialized countries such as Germany, where HEV infections are generally caused by consumption of HEV contaminated pork and transfusion of HEV positive blood, routine screenings for blood resource and pork can be put into application. In contrast, for those developing countries such as India where HEV are transmitted to humans primarily through sewage contaminated water, assurance in clean water supply should be their primary task. Global vaccination against HEV is also a possible solution in preventing the spread of viral infections. Although effective vaccines have been invented it is only approved for use in China but no other parts of the world. Efforts should be undertaken to make HEV vaccines widely accessible to all parts of the world, both industrialized and developing countries, under affordable costs. This would be a major tool for containing the spread of HEV and preventing the severe and sometimes fatal disease.

\section{ACKNOWLEDGEMENTS}

Special thanks to Professor Alexander Ploss from Princeton University for the academic support of this article and to the CIS program for offering this opportunity of studying and doing research upon this subject. 


\section{CONFLICTS OF INTEREST}

The authors declare no conflicts of interest regarding the publication of this paper.

\section{REFERENCES}

1. Ding, N. (2017) Hepatitis E Is a Liver Disease Caused by Hepatitis E Virus (HEV) Transmitted through the Faecal-Oral Route.

http://www.drhealthtopics.com/hepatitis-e-liver-disease-caused-hepatitis-e-virus-hev-transmitted-faecal-oral-ro $\underline{\text { ute/ }}$

2. Kadir, S.M., Saleem-ur-Rehman, K., Benetou, K., Ahmad, D. and Abdullah, A. (2016) Ten Years of Disease Surveillance in Kashmir, India under Integrated Disease Surveillance Programme (IDSP) during 2006-2016.

https://www.amhsr.org/articles/ten-years-of-disease-surveillance-in-kashmir-india-under-integrated-disease-su rveillance-programme-idsp-during-20062016-4186.html

3. Leonad, N. (2016) Mikhail Balayan and the Bizarre Discovery of Hepatitis E Virus. https://norkinvirology.wordpress.com/2016/05/03/mikhail-balayan-and-the-bizarre-discovery-of-hepatitis-e-vir us/

4. WHO, K. (2017) Hepatitis E. http://www.who.int/news-room/fact-sheets/detail/hepatitis-e

5. Daniel, C. and Olender, S. (2018) How Can You Prevent Diseases Spread by the Fecal-Oral Route? https://www.verywellhealth.com/what-is-the-fecal-oral-route-1760046

6. Kamar, N., Izopet, J., Pavio, N., Aggarwal, R., Labrique, A., Wedemeyer, H. and Dalton, H.R. (2017) Hepatitis E Virus Infection. https://www.ncbi.nlm.nih.gov/pubmed/29154369

7. de Alencar Arrais Guerra, J.A., Kampa, K.C., Morsoletto, D.G., Junior, A.P. and Ivantes, C.A. (2017) Hepatitis E: A Literature Review. https://www.ncbi.nlm.nih.gov/pmc/articles/PMC5719195/

8. Pelosi, E. and Clarke, I. (2008) Hepatitis E: A Complex and Global Disease. https://www.ncbi.nlm.nih.gov/pmc/articles/PMC3167588/

9. Ding, Q., Heller, B., Capuccino, J.M., Song, B., Nimgaonkar, I., Hrebikova, G., et al. (2017) Hepatitis E Virus ORF3 Is a Functional Ion Channel Required for Release of Infectious Particles.

http://www.pnas.org/content/114/5/1147

10. Cao, D. and Meng, X. (2012) Molecular Biology and Replication of Hepatitis E Virus.

https://www.nature.com/articles/emi20127

11. Song, Y. (2010) Studies of Hepatitis E Virus Genotypes.

https://www.ncbi.nlm.nih.gov/pmc/articles/PMC3028963/

12. Pischke, S., Behrendt, P., Bock, C., Jilg, W., Manns, M. and Wedemeyer, H. (2014). Hepatitis E in Germany-An Under-Reported Infectious Disease. https://www.ncbi.nlm.nih.gov/pmc/articles/PMC4174681/

13. Cornelia, A., Alexander, W., Helga, M., Marco, K., Heinz, E. and Georg, P. (2009) High HEV Presence in Four Different Wild Boar Populations in East and West Germany.

https://www.sciencedirect.com/science/article/pii/S0378113509003174

14. Szabo, K. and ReimarJohneb, R. (2015) Detection of Hepatitis E Virus RNA in Raw Sausages and Liver Sausages from Retail in Germany Using an Optimized Method. https://www.sciencedirect.com/science/article/pii/S0168160515301264

15. Krumbholz, A., Joel, S., Dremsek, P., et al. (2014) Seroprevalence of Hepatitis E Virus (HEV) in Humans Living in High Pig Density Areas of Germany. Medical Microbiology and Immunology, 203, 273-282.

https://link.springer.com/article/10.1007/s00430-014-0336-3 
16. Bettinger, D.E.H.H., Kappert, O.M. and Wenzel, J. (2014) Transfusion-Transmitted Hepatitis E in Germany, 2013. Eurosurveillance, 19, pii: 20812.

https://www.eurosurveillance.org/content/10.2807/1560-7917.ES2014.19.21.20812

17. Vollmer, T., Diekmann, J., Johne, R., Eberhardt, M., Knabbe, C. and Dreier, J. (2012) Novel Approach for Detection of Hepatitis E Virus Infection in German Blood Donors. Journal of Clinical Microbiology, 50, 2708-2713.

18. Vollmer, T., Diekmann, J., Eberhardt, M., Knabbe, C. and Dreier, J. (2016) Hepatitis E in Blood Donors: Investigation of the Natural Course of Asymptomatic Infection, Germany, 2011. Eurosurveillance, 21.

https://www.ncbi.nlm.nih.gov/pubmed/27608433

19. Jain, P., Prakash, S., Gupta, S., Singh, K.P., Shrivastava, S., Singh, D.D. and Jain, A. (2013) Prevalence of Hepatitis A Virus, Hepatitis B Virus, Hepatitis C Virus, Hepatitis D Virus and Hepatitis E Virus as Causes of Acute Viral Hepatitis in North India: A Hospital Based Study. Indian Journal of Medical Microbiology, 31, 261-265. https://www.ncbi.nlm.nih.gov/pubmed/23883712/

20. Tnn, N. (2013) Around 80\% of Sewage in Indian Cities Flows into Water System.

https://timesofindia.indiatimes.com/home/environment/pollution/Around-80-of-sewage-in-Indian-cities-flows -into-water-systems/articleshow/18804660.cms

21. Ippagunta, S., Sharma, B. and Aggarwal, R. (2007) Presence of Hepatitis E Virus in Sewage in Northern India: Frequency and Seasonal Pattern. Journal of Medical Virology, 79, 1827-1831. https://onlinelibrary.wiley.com/doi/abs/10.1002/jmv.21017

22. Helen, W. (2017) Seroprevalence and Risk Factors Associated with Hepatitis E Virus Infections among People and Pigs in Tibet, China. Acta Tropica, 172, 102-106. https://www.sciencedirect.com/science/article/pii/S0001706X17302875?via=ihub

23. Jia, Z., Yi, Y., Liu, J., Cao, J., Zhang, Y., Tian, R., Bi, S., et al. (2014) Epidemiology of Hepatitis E Virus in China: Results from the Third National Viral Hepatitis Prevalence Survey, 2005-2006. PLoS ONE, 9, e110837. https://www.ncbi.nlm.nih.gov/pmc/articles/PMC4215996/ https://doi.org/10.1371/journal.pone.0110837

24. Dong, C., Dai, X., Liang, J., Dong, M. and Meng, J. (2012) Seroprevalence of Hepatitis E Virus Varies Considerably among Chinese Provinces. Hepatitis Monthly, 12, 386-390. http://hepatmon.com/en/articles/70416.html

25. Worm, H.C. and Wirnsberger, G. (2004) Hepatitis E Vaccines: Progress and Prospects. Drugs, 64, 1517-1531. https://doi.org/10.2165/00003495-200464140-00002

26. Ahmad, I., Holla, R.P. and Jameel, S. (2011) Molecular Virology of Hepatitis E Virus. Virus Research, 161, 47-58. https://doi.org/10.1016/j.virusres.2011.02.011

27. Makar, G. (2007) Safety and Efficacy of a Recombinant Hepatitis E Vaccine. The New England Journal of Medicine, 356, 895-903. https://doi.org/10.1016/S0739-5930(08)70198-3

28. Zhang, J. (2015) Long-Term Efficacy of a Hepatitis E Vaccine. New England Journal of Medicine, 372, 1478-1478. https://doi.org/10.1056/NEJMoa1406011

29. Wut, T. (2015) Zhongguo Wuganyimian - Weiyufang Dailai Xiwang. http://news.medlive.cn/all/info-progress/show-45099_35.html

30. Us, C. (2018) Vaccines for Children Program (VFC). https://www.cdc.gov/vaccines/programs/vfc/awardees/vaccine-management/price-list/index.html

31. (2018) Germany GDP per Capita PPP 1990-2018. https://tradingeconomics.com/germany/gdp-per-capita-ppp 
32. Vivek, R., Zachariah, U., Ramachandran, J., Eapen, C., Rajan, D. and Kang, G. (2013) Characterization of Hepatitis E Virus from Sporadic Hepatitis Cases and Sewage Samples from Vellore, South India. Transactions of the Royal Society of Tropical Medicine and Hygiene, 107, 363-367. https://doi.org/10.1093/trstmh/trt030 https://www.ncbi.nlm.nih.gov/pmc/articles/PMC5454524/

33. (2018) Deguo Juanxian Qiguan Renshu Chuangxindi - Xinhuawang. http://www.xinhuanet.com/world/2018-01/16/c_1122266247.htm

34. (2018) 2018 Nian Zhongguo Qiguan Juanxian Youwang Tupo 6000 li - Xinhuawang. http://www.xinhuanet.com/politics/2018-07/14/c_129913433.htm

35. Domanović, D., Tedder, R., Blümel, J., Zaaijer, H., Gallian, P., Niederhauser, C., Hewitt, P., et al. (2017) Hepatitis E and Blood Donation Safety in Selected European Countries: A Shift to Screening? Eurosurveillance, 22, 30514. https://www.ncbi.nlm.nih.gov/pmc/articles/PMC5404480/ https://doi.org/10.2807/1560-7917.ES.2017.22.16.30514

36. Guo, Q., Yan, Q., Xiong, J., Ge, S., Shih, J., Ng, M., Xia, N., et al. (2009) Prevalence of Hepatitis E Virus in Chinese Blood Donors. Journal of Clinical Microbiology, 48, 317-318.

https://www.ncbi.nlm.nih.gov/pmc/articles/PMC2812292/

https://doi.org/10.1128/JCM.01466-09

37. Gajjar, M., Bhatnagar, N., Sonani, R., Gupta, S. and Patel, T. (2014) Hepatitis E Seroprevalence among Blood Donors: A Pilot Study from Western India. Asian Journal of Transfusion Science, 8, 29-31.

https://www.ncbi.nlm.nih.gov/pmc/articles/PMC3943141/ https://doi.org/10.4103/0973-6247.126685

38. Wang, M., Yin, Y., He, M. and Liu, Y. (2016) Acute, Recent and Past HEV Infection among Voluntary Blood Donors in China: A Systematic Review and Meta-Analysis. PLoS ONE, 11, e0161089.

http://journals.plos.org/plosone/article?id=10.1371\%2Fjournal.pone.0161089 https://doi.org/10.1371/journal.pone.0161089

39. Wen, G., Chen, C., Song, X., Tang, Z., Ji, W., Wang, S., Xia, N., et al. (2018) Long-Term HEV Carriers without Antibody Seroconversion among Eligible Immunocompetent Blood Donors. Emerging Microbes \& Infections, 7, 125. https://www.ncbi.nlm.nih.gov/pmc/articles/PMC6033859/ https://doi.org/10.1038/s41426-018-0125-y

40. BioPharm, W. (2013) HEV-IgM Testing Paper Box. http://www.ystwt.com/product/276519599

41. BioPharm, W. (2015) Hepatitis E Virus IGM Detection Reagent. http://www.wondfo.com.cn/product-165.html

42. (2017) Ribavirin-FDA Prescribing Information, Side Effects and Uses. https://www.drugs.com/pro/ribavirin.html

43. Goldszmid, R., Dzutsev, A. and Trinchieri, G. (2014) Host Immune Response to Infection and Cancer: Unexpected Commonalities. Cell Host \& Microbe, 15, 295-305.

https://www.ncbi.nlm.nih.gov/pmc/articles/PMC3996827/

https://doi.org/10.1016/j.chom.2014.02.003

44. Kamar, N., et al. (2014) Ribavirin for Chronic Hepatitis E Virus Infection in Transplant Recipients. The New England Journal of Medicine, 370, 1111-1120. https://www.nejm.org/doi/full/10.1056/NEJMoa1215246 https://doi.org/10.1056/NEJMoa1215246

45. Yeh, M., Hsieh, M., Huang, C., Huang, C., Hsieh, M., Huang, J., Yu, M., et al. (2016) Long-Term Efficacy of Peg-Interferon/Ribavirin with and without Lamivudine Therapy for HBeAg-Positive Hepatitis B and C Dual Infection. Journal of Gastroenterology and Hepatology, 31, 835-841. 
https://www.ncbi.nlm.nih.gov/pubmed/26478984

https://doi.org/10.1111/jgh.13203

46. Kamar, N., Lhomme, S., Peron, O.M., Alric, L. and Izopet, J. (2016) Treatment of HEV Infection in Patients with a Solid-Organ Transplant and Chronic Hepatitis. Viruses, 8, 222.

http://www.mdpi.com/1999-4915/8/8/222/htm

https://doi.org/10.3390/v8080222

47. (2018) Ribavirin Side Effects in Detail-Drugs.com.

https://www.drugs.com/sfx/ribavirin-side-effects.html 